See discussions, stats, and author profiles for this publication at: https://www.researchgate.net/publication/293646824

\title{
The effects of acupuncture on polycystic ovary syndrome: A systematic review and meta-analysis
}

Article in European Journal of Integrative Medicine · February 2016

DOI: 10.1016/j.eujim.2016.02.001

CITATIONS

5

14 authors, including:

Mio (Xiaoyang Hu)

University of Southampton

43 PUBLICATIONS 124 CITATIONS

SEE PROFILE

(8) Jisheng Han

(1) Peking University

536 PUBLICATIONS 12,976 CITATIONS

SEE PROFILE

Some of the authors of this publication are also working on these related projects:

Project Women and health View project

Project Quality of life in childless older people View project
READS

259

John A Barry

University College London

60 PUBLICATIONS 674 CITATIONS

SEE PROFILE 


\section{Accepted Manuscript}

Title: The effects of acupuncture on polycystic ovary syndrome: A systematic review and meta-analysis

Author: Fan Qu Yan Wu Xiao-Yang Hu John A. Barry Jue Zhou Fang-Fang Wang Ying-Hui Ye Rong Zhang Song-Ping Han Ji-Sheng Han Rong Li Malcolm B. Taw Paul J. Hardiman

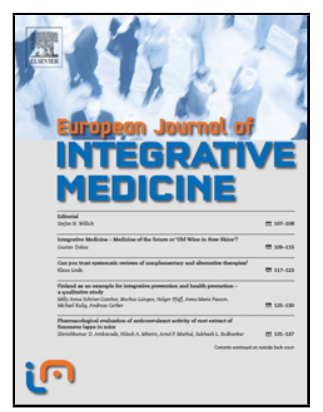
Nicola Robinson

PII: S1876-3820(16)30008-7

DOI: $\quad$ http://dx.doi.org/doi:10.1016/j.eujim.2016.02.001

Reference: $\quad$ EUJIM 505

To appear in:

Received date: $\quad$ 28-10-2015

Revised date: $\quad 1-2-2016$

Accepted date: $\quad 1-2-2016$

Please cite this article as: Qu Fan, Wu Yan, Hu Xiao-Yang, Barry John A, Zhou Jue, Wang Fang-Fang, Ye Ying-Hui, Zhang Rong, Han Song-Ping, Han Ji-Sheng, Li Rong, Taw Malcolm B, Hardiman Paul J, Robinson Nicola.The effects of acupuncture on polycystic ovary syndrome: A systematic review and meta-analysis.European Journal of Integrative Medicine http://dx.doi.org/10.1016/j.eujim.2016.02.001

This is a PDF file of an unedited manuscript that has been accepted for publication. As a service to our customers we are providing this early version of the manuscript. The manuscript will undergo copyediting, typesetting, and review of the resulting proof before it is published in its final form. Please note that during the production process errors may be discovered which could affect the content, and all legal disclaimers that apply to the journal pertain. 
The effects of acupuncture on polycystic ovary syndrome:

A systematic review and meta-analysis

Fan $\mathrm{Qu}^{\mathrm{a}}{ }^{*}$, Yan $\mathrm{Wu}^{\mathrm{a}}{ }^{*}$, Xiao-Yang $\mathrm{Hu}^{\mathrm{b}^{*}}$, John A. Barry ${ }^{\mathrm{c}}$, Jue Zhou ${ }^{\mathrm{d}}$, Fang-Fang Wang ${ }^{\mathrm{a}}$, Ying-Hui Ye ${ }^{\mathrm{a}}$, Rong Zhang ${ }^{\mathrm{e}}$, Song-Ping Han ${ }^{\mathrm{e}}$, Ji-Sheng Han ${ }^{\mathrm{e}}$, Rong $\mathrm{Li}^{\mathrm{f}}$, Malcolm B. Taw ${ }^{\mathrm{g}}$, Paul J. Hardiman ${ }^{\mathrm{c}}$, Nicola Robinson ${ }^{\mathrm{b} *}$

a Women's Hospital, School of Medicine, Zhejiang University, Hangzhou, Zhejiang, 310006, China

b School of Health and Social Care, London South Bank University, London, SE1 0AA, UK.

c Institute for Women's Health, University College London Medical School, London, NW3 2PF, UK.

d College of Food Science and Biotechnology, Zhejiang Gongshang University, Hangzhou, Zhejiang, 310012, China

e Neuroscience Research Institute \& Department of Neurobiology, School of Basic Medical Sciences, Peking University, Beijing, 100083, China

f Peking University Third Hospital, Beijing, 100191, China

g UCLA Center for East-West Medicine - Westlake Village, UCLA Department of Medicine, David Geffen School of Medicine, UCLA, Westlake Village, CA 91361, U.S.A.

* The authors contributed equally to the study 


\title{
** Corresponding author
}

Address all correspondence and requests for reprints to: Professor Nicola Robinson, School of Health and Social Care, London South Bank University, 103 Borough Road, London SE1 0AA, UK. Tel: $\quad+44$ (0) 207815 7940, Fax: +44 (0) 207815 8490, E-mail: nicky.robinson@1sbu.ac.uk

Running title: acupuncture for PCOS

\begin{abstract}
Introduction: To assess the clinical effectiveness of acupuncture in treating polycystic ovarian syndrome (PCOS).

Methods: A systematic review and meta-analysis was conducted of RCTs that compared either acupuncture with no/sham (placebo) acupuncture or a certain therapy with acupuncture added in the treatment of PCOS. Measures of treatment effectiveness were the pooled odds ratios (OR) for women with PCOS having acupuncture compared with women in the control group for the recovery of menstrual cycles, standardized mean difference (SMD) for body mass index (BMI), fasting insulin (FINS), fasting plasma glucose (FPG), luteinizing hormone ( $\mathrm{LH})$, follicle stimulating hormone (FSH), and the ratio of $\mathrm{LH} / \mathrm{FSH}$.

Results: A total of nine RCTs (531 women) met criteria for inclusion into the systematic review. Using the random effects model, pooling of the effect estimates from all RCTs showed recovery of menstrual cycles $(\mathrm{OR}=0.20,95 \% \mathrm{CI}$ : 0.09 to $0.41, \mathrm{P}<0.01)$, BMI $(\mathrm{SMD}=-0.63,95 \% \mathrm{CI}:-1.04$ to $-0.21, \mathrm{P}=0.04)$, and $\mathrm{LH}(\mathrm{SMD}=-0.39,95 \% \mathrm{CI}:-0.65$
\end{abstract}


to $-0.12, \mathrm{P}<0.01)$ which favored the acupuncture group. No significant differences were observed for FINS, FPG, FSH and the ratio of LH/FSH between acupuncture and control groups $(\mathrm{P}>0.05)$.

Conclusions: Acupuncture appears to significantly improve the recovery of the menstrual cycles and decrease the levels of BMI and LH in women with PCOS. However, the findings should be interpreted with caution due to the limited methodological quality of included RCTs.

Keywords: Acupuncture; Polycystic ovary syndrome (PCOS); Meta-analysis.

\section{Introduction}

Polycystic ovary syndrome (PCOS) is recognized as one of the most common endocrine and metabolic disorders in women, which has a prevalence of 6 to $15 \%$ in unselected reproductive-aged women $[1,2]$. The PCOS patients often need pharmacological treatment over a long period of time[3, 4]. Although oral contraceptives are the most common therapy to effectively alleviate hirsutism and acne of patients with PCOS, they can adversely affect glucose tolerance, coagulability and fertility [5].

Acupuncture, through stimulating specific points on the body, has been used to treat gynecological disorders for thousands of years [6]. Acupuncture is sometimes chosen by women with PCOS as an alternative to pharmacotherapy, with some choosing to use it as an adjunct while undergoing infertility treatment [7]. We herein provide a systematic review and meta-analysis of the available literature to assess the effectiveness of acupuncture in the treatment of PCOS. 


\section{Methods}

\section{Eligibility criteria}

Randomized controlled trials (RCTs) comparing either acupuncture with no/sham (placebo) acupuncture or a certain therapy with acupuncture added in the treatment of PCOS were included. "Acupuncture" was defined as traditional needling, auricular acupuncture, electro-acupuncture (EA), auricular acupressure, or warm needling acupuncture. PCOS was diagnosed according to the European Society for Human Reproduction and Embryology (ESHRE) and American Society for Reproductive Medicine (ASRM) sponsored PCOS Consensus Workshop criteria (the Rotterdam criteria) [8]. For inclusion, at least one of the following outcomes had to be available for extraction: recovery of menstrual cycles, body mass index (BMI), fasting insulin (FINS), fasting plasma glucose (FPG), luteinizing hormone (LH), follicle stimulating hormone (FSH), the ratio of LH/FSH and testosterone (T). The numbers of events, participants in each group, mean and standard deviation (SD) for continuous data had to be clearly defined. Case reports, reviews, animal experiments, non-randomized controlled trials and studies comparing the combination of acupuncture and another therapy with a third therapy were excluded.

\section{Search strategy}

A systematic literature search was performed using the following databases: MEDLINE, EMBASE, SCISEARCH, the Cochrane Menstrual Disorders and Subfertility Group trials register, China Academic Journal Electronic full text Database in China National Knowledge Infrastructure, Wanfang Database, Index to Chinese Periodical Literature, and the International Standard Randomized Controlled Trial Number (ISRCTN) Register and 
meta-register for randomized controlled trials (mRCT). All the databases were searched from their inception to May 2015. The reference lists of the relevant primary and review articles were examined to identify cited articles that were not captured by electronic searches. No restrictions of language or publication type were placed on the searches.

The following keywords were searched: "polycystic ovary syndrome" or "polycystic ovary" or "ovary polycystic disease" or "PCOS" or "polycystic ovary morphology" or "oligo-amenorrhea" or "oligoamenorrhea" or "oligoanovulatory" or "oligohypomenorrhea" or "amenorrhea" or "amenorrhoea" or "hirsutism" AND "acupoint" or "acupressure" or "acupressure-acupuncture therapy" or "acupuncture" or "electro-acupuncture" or "electroacupuncture" or "moxibustion" or "Tui $\mathrm{Na"} \mathrm{or}$ " traditional medicine" or "traditional Chinese medicine" or "traditional Chinese medicine combined with western medicine". All search terms were back translated into Chinese terms in order to conduct the searches in Chinese databases.

\section{Study selection}

Two reviewers independently scrutinized titles and abstracts from the electronic searches (F. Q. and Y.W.), and full manuscripts of all citations that were likely to meet the predefined inclusion criteria were obtained. The final inclusion or exclusion decisions were made on examination of the full manuscripts. In cases of duplicate publication, the most recent and complete versions were selected. Any disagreements about inclusion were resolved by discussion or arbitration by a third reviewer (X.H.).

\section{Quality assessment and data extraction}

Two independent reviewers (F.Q. and Y.W.) assessed the risk of bias with the criteria in

the Cochrane Handbook for Systematic Reviews of Interventions 5.1.0 
(http://community.cochrane.org/handbook). Any disagreements between the reviewers were resolved by discussion and arbitration by a third reviewer (X.H.).

Two reviewers (F. Q. and Y.W.) extracted data from all eligible articles independently. Data that was collected included the year of publication, study design, patient characteristics, sample size and relevant outcome measurements. The safety of using acupuncture and other therapies was also assessed by exploring the description of side effects in the included RCTs.

\section{Statistical analysis}

The results were pooled and expressed as odds ratio (OR) or standard mean difference (SMD) with 95\% confidence interval (CI). Heterogeneity of treatment effects was evaluated graphically using a forest plot and a chi-square test. A value greater than $50 \%$ was considered as substantial heterogeneity. In the meta-analysis, a random effects model was used for the encountered heterogeneity of the trials' characteristics and populations studied. A sensitivity analysis was conducted to examine the effect of excluding each study. The statistical analysis was performed with STATA 12.0 software (StataCorp, College Station, USA).

\section{Results}

\section{Main study characteristics}

A total number of 173 citations were obtained from the electronic searches and examination of the reference lists of primary and review articles, of which, 75 were selected for retrieval (as shown in Figure 1). We followed the protocol of "Preferred Reporting Items for Systematic Review and Meta-Analyses (PRISMA)" in the flow diagram. The process of literature identification and selection is given in the flowchart 
(Figure 1). Of the 75 full manuscripts examined, nine trials including a total of 531 women met inclusion criteria. All nine RCTs were included in the meta-analysis. The quality evaluation and summary of the characteristics of the included trials are identified in Table 1 and Table 2 respectively.

\section{Measurement of risk of bias}

The risk of bias was measured for each of the nine studies included. Although all the trials mentioned randomization, only five studies described their specific randomization strategies [9-14].

Two of the included RCTs had a high risk of selection bias as the sequence was generated by the registration order $[9,12]$. Seven trials had a high risk of performance bias as the sham acupuncture was not used as a control in the trials $[9,12,14-19]$. The reporting bias was unclear in eight RCTs because we were unable to obtain access to the protocol and verify their primary/secondary outcome measurements [9-12, 14-19]. The summary of the assessment of risk of bias was shown as Supplemental Table 1.

\section{Recovery of menstrual cycles}

For the recovery of menstrual cycles, data were available for 5 of the 9 included trials $(\mathrm{N}$ $=247)[9,12-14,18]$. Using the random effects model, no significant statistical heterogeneity between the studies was found $\left(\mathrm{I}^{2}=0.0 \%, \mathrm{P}=0.93\right)$. Pooling of the effect estimates from all of the five trials showed significant efficacy $(\mathrm{P}<0.01)$ of acupuncture in improving the recovery of menstrual cycles of women with PCOS $(\mathrm{OR}=0.20,95 \% \mathrm{CI}$ : 0.09 to 0.41 , Figure $2 \mathrm{~A}$ ). The sensitivity analysis showed that the significance of the pooled OR derived from the 5 RCTs was not excessively influenced by any single study. 


\section{Body Mass Index (BMI)}

For BMI, data were available from 3 of the 9 included trials $(\mathrm{N}=155)[13,15,19]$. Using the random effects model, the statistical heterogeneity between the studies was not significant $\left(\mathrm{I}^{2}=29.5 \%, \mathrm{P}=0.24\right)$. The pooled $\mathrm{SMD}$ of $\mathrm{BMI}$ showed significant effectiveness $(\mathrm{P}=0.04)$ of acupuncture in reducing BMI of the women with PCOS (SMD $=-0.63,95 \% \mathrm{CI}:-1.04$ to -0.21 , Figure $2 \mathrm{~B})$. The sensitivity analysis of BMI found that one RCT [15] affected the pooled SMD. When the study was removed, the pooled SMD was not significant $(\mathrm{SMD}=-0.66,95 \% \mathrm{CI}:-1.47$ to 0.14$)$.

\section{Fasting Insulin (FINS) and Fasting Plasma Glucose (FPG)}

For FINS and FPG, the data were available from the same three trials as BMI $(\mathrm{N}=155)$ $[13,15,19]$. Using the random effects model, no significant statistical heterogeneity for FINS and FPG was found $\left(\mathrm{I}^{2}=0.0 \%\right.$ and $0.0 \%, \mathrm{P}=0.83$ and 0.61 , respectively). Pooling of the results from all three trials showed no significant difference on FINS and FPG between the acupuncture and the control groups ( $\mathrm{SMD}=0.11,95 \% \mathrm{CI}$ : -0.21 to 0.43 ; $\mathrm{SMD}=0.02,95 \% \mathrm{CI}:-0.30$ to 0.34 , respectively, Figure $2 \mathrm{C}$ and $\mathrm{D})$. The pooled SMD derived from the 3 RCTs was not excessively influenced by any single study.

\section{Hormone levels}

For the hormone levels, the data of LH and FSH were available from five trials $(\mathrm{N}=302)$ $[10,11,13-17]$. Using the random effects model, no significant statistical heterogeneity for $\mathrm{LH}$ and FSH was found $\left(\mathrm{I}^{2}=20.9 \%\right.$ and $20.7 \%, \mathrm{P}=0.28$ and 0.28 , respectively $)$. Pooling of the results from all five trials showed significant efficacy $(\mathrm{P}=0.02)$ of acupuncture in regulating serum LH levels of the women with PCOS (SMD $=-0.39,95 \%$ CI: -0.65 to -0.12 , Figure $3 \mathrm{~A}$ ), however, there was no significant difference for serum 
FSH levels between acupuncture and the control groups (SMD $=-0.11,95 \% \mathrm{CI}:-0.37$ to 0.15 , Figure 3B). Data of the ratio of $\mathrm{LH} / \mathrm{FSH}$ were available from 5 of the 9 included trials $(\mathrm{N}=282)[10,11,13,14,16,17,19]$. Using the random effects model, no significant statistical heterogeneity was found $\left(\mathrm{I}^{2}=0.0 \%, \mathrm{P}=0.74\right)$. Pooling of the results from all five trials showed no significant difference in the ratio of LH/FSH between the acupuncture and control groups (SMD $=-0.20,95 \%$ CI: -0.43 to 0.04 Figure $3 \mathrm{C}$ ). Sensitivity analysis showed that the pooled SMDs of LH, FSH and LH/FSH were not excessively influenced by any single trial.

As hyperandrogenism is an important feature of PCOS, testosterone should be included in the meta-analysis. Testosterone was measured in five of the included RCTs [13-17, 19], however, four of them failed to clarify whether it was total testosterone or free testosterone $[14-17,19]$. Moreover, the unit of testosterone was not mentioned in one of them [19] and in the other four trials, the units of testosterone were different. As such, it was determined that testosterone is not an appropriate outcome measurement for the present meta-analysis. However, four of these five studies showed acupuncture could significantly decrease the serum level of testosterone in PCOS women [14-17, 19].

\section{The safety of acupuncture in treating PCOS}

Unfortunately, none of these nine included RCTs assessed the adverse effects of acupuncture or other therapies during the research period.

\section{Discussion}

\section{Summary of evidence}

Based on the findings from the present systematic review and meta-analysis, acupuncture appeared to significantly improve the recovery of menstrual cycles and lower BMI and 
LH levels in women with PCOS; however, there was no evidence to suggest that acupuncture could improve the levels of FINS, FPG, FSH or the ratio of LH/FSH of the PCOS patients.

For clinical trials, a detailed description of study design is critical to evaluate the evidence. In seven of the included studies, the methods of allocation concealment and blinding were not mentioned [9, 12, 14-19], which may lead to selection and performance biases. The randomization strategies were inadequate in six of the nine studies $[9,12$, 15-19], which could also lead to selection bias. In addition, the maximum sample size was no larger than 100 , and the minimum was only 15 . Given the low methodological quality of these studies, conclusions about the clinical effectiveness of acupuncture for treating PCOS should be interpreted with caution.

In the present meta-analysis, seven single-center trials were conducted in China $[9,12$, 14-19]. Chinese patients have a preference for acupuncture as a treatment, which may enhance the placebo effect on them. It is also more likely that acupuncture research conducted in Asian countries have positive outcomes [20].

Recall bias may also exist, as all the included trials failed to provide detailed information of patients who were loss to follow-up. Moreover, some high-quality RCTs are not included in the present meta-analysis due to unavailable data[21, 22].

The results of the bias assessment in the present meta-analysis, the majority of included trials were determined to have "unclear" or "high" risks of bias in the domains of allocation concealment, blinding and random sequence generation. These methodological domains should be assured in future RCTs. In addition, multi-center trials with larger samples should also be taken into consideration. 
In the process of reviewing articles, we found that most Chinese studies only evaluated the recovery of menstrual cycles and measured the hormone levels, while other studies had more outcome measurements. As PCOS is a multisystem disease with various clinical symptoms, more outcomes, such as Ferriman-Gallwey score, insulin sensitivity, and blood lipids, should also be measured. The quality of life among patients with PCOS has also received more attention in recent years and many scales, such as the Montgomery Åsberg Depression Rating Scale (MADRS-S), Brief Scale for Anxiety (BSA-S), Swedish Short-Form 36 (SF-36), and PCOS Questionnaire (PCOSQ) scores have been used to evaluate this [23]. These patient-centered outcome measures should also be used to evaluate the effect of acupuncture on PCOS, in conjunction with conventional measures.

During the process of data extraction, we found that continuous data in some high-quality studies were not shown as mean and SD; and various units of some indicators were used in different trials. These problems limited the number of included studies and influenced the statistical power in the current meta-analysis. Therefore, the integrity of data need to be ensured in further studies.

In our review, only two RCTs $[10,11,13]$ adhered to STRICTA, a series of guidelines to provide authors a way to structure their reports of acupuncture interventions with a minimum set of items using a checklist. According to the STRICTA, acupuncture rationale, details of needling, treatment regimen, other components of treatment, practitioner background, and the control or comparator interventions should all be reported in the methods section [24].

Another limitation in this review was that all trials comparing acupuncture and other 
inventions were included in the meta-analysis, irrespective of the design of control group. This was due to the variety of PCOS treatment and an inadequate number available in same comparison. However, the heterogeneity is acceptable in the meta-analysis, as all the $\mathrm{I}^{2}$ is less than $30 \%$.

For any clinical intervention, the safety of patients should be monitored critically and reported as part of the trial procedures. None of the trials identified in this review provided information on records of adverse effects during the research period. Future RCTs should incorporate reporting of adverse effects with detailed information of receiving acupuncture and any treatment related events.

\section{Conclusion}

From our meta-analysis, acupuncture appears to significantly improve the recovery of menstrual cycles and lower BMI and LH levels in women with PCOS. However, given the limited methodological quality of included RCTs, these findings should be interpreted with caution.

\section{Conflicts of interest}

No competing financial interests exist.

\section{Acknowledgements}

This work was supported by China Scholarship Council (No. 201308330139) and the Ministry of Health Special Fund of China (No. 201302013).

\section{References}

[1] Klimczak D, Szlendak-Sauer K, Radowicki S. Depression in relation to biochemical parameters and age in women with polycystic ovary syndrome. European Journal of Obstetrics, Gynecology, and Reproductive Biology. 2015;184:43-7.

[2] Stefanaki C, Bacopoulou F, Livadas S, Kandaraki A, Karachalios A, Chrousos GP, et al. Impact of a mindfulness stress management program on stress, anxiety, depression and quality of life in women with polycystic ovary syndrome: a randomized controlled trial. 
Stress. 2015;18:57-66.

[3] Ndefo UA, Eaton A, Green MR. Polycystic ovary syndrome: a review of treatment options with a focus on pharmacological approaches. P \& $\mathrm{T}$ : a peer-reviewed journal for formulary management. 2013;38:336-55.

[4] Norman RJ, Dewailly D, Legro RS, Hickey TE. Polycystic ovary syndrome. Lancet. 2007;370:685-97.

[5] Lanham MS, Lebovic DI, Domino SE. Contemporary medical therapy for polycystic ovary syndrome. International journal of gynaecology and obstetrics: the official organ of the International Federation of Gynaecology and Obstetrics. 2006;95:236-41.

[6] Barry JA, Kuczmierczyk AR, Hardiman PJ. Reporting the rates of depression in polycystic ovary syndrome (PCOS). The Journal of Sexual Medicine. 2014;11:1882-3.

[7] Stener-Victorin E, Jedel E, Manneras L. Acupuncture in polycystic ovary syndrome: current experimental and clinical evidence. Journal of Neuroendocrinology. 2008;20:290-8.

[8] Consensus TREA-SP, Group W. Revised 2003 consensus on diagnostic criteria and long-term health risks related to polycystic ovary syndrome. Fertility and Sterility. 2004;81:19-25.

[9] Wang H, Gu SJ. Clinical Observation of Acupuncture Combined with Chinese Herb in the Treatment of PCOS with Infertility. Journal of Hubei University of Chinese Medicine. 2013;15:57-8.

[10] Franasiak J, Young SL, Williams CD, Pastore LM. Longitudinal anti-mullerian hormone in women with polycystic ovary syndrome: an acupuncture randomized clinical trial. Evidence-based Complementary and Alternative Medicine. 2012;2012:973712.

[11] Pastore LM, Williams CD, Jenkins J, Patrie JT. True and sham acupuncture produced similar frequency of ovulation and improved LH to FSH ratios in women with polycystic ovary syndrome. The Journal of Clinical Endocrinology and Metabolism. 2011;96:3143-50.

[12] Li B, Han JR. Clinical Observation of Acupuncture Combined with Chinese Herb in the Treatment of PCOS. Journal of Clinical Acupuncture and Moxibustion. 2010;26:20-1. [13] Stener-Victorin E, Jedel E, Janson PO, Sverrisdottir YB. Low-frequency electroacupuncture and physical exercise decrease high muscle sympathetic nerve activity in polycystic ovary syndrome. American Journal of Physiology Regulatory, Integrative and Comparative Physiology. 2009;297:R387-95.

[14] Shi Y, Feng HJ, Liu HR, Zhu D. [Observation on therapeutic effect of acupuncture combined with chinese herbs on polycystic ovary syndrome of kidney deficiency and phlegm stasis type]. Zhongguo Zhenjiu. 2009;29:99-102.

[15] Liu XX, M. LY, Li XY, Liang RW, Pan ML, Liu XX. Combination of acupuncture and medicine in regulating disorders of PCOS women. Chinese Journal of Medicinal Guide. 2012;14:112-4.

[16] Liang RW, Liu XX, Li XY, Liu YM. The Effects of Acupoint Block, Needling and Assisted Reproductive Technique on the Level of Hormone in Infertile Patients with PCOS. Western Journal of Traditional Chinese Medicine. 2013;26:98-9.

[17] Liang RW, Liu XX, Li XY, Liu YM. Effect of Points Acupuncture and Closeness Combined with Assisted Reproductive Technology on Ocytes Maturity of PCOS Infertility Patients. Journal of Clinical Acupuncture and Moxibustion. 2013;29:17-9.

[18] Lin SH. Clinical Observation of Acupuncture in the Treatment of PCOS. 
Asia-Pacific Traditional Medicine. 2014;10:89-90.

[19] Zhao Y, Hu LS, Wang CH, Wang XP, Du XH, Shao H, et al. The Study of Treatment with Compoud Acupuncture-Metformin on the Outcome of Ovulation Induction with Chloramiphene in the Patients with PCOS. Jiangxi Medical Journal. 2007;42:1089-92.

[20] Acmaz G, Albayrak E, Acmaz B, Baser M, Soyak M, Zararsiz G, et al. Level of anxiety, depression, self-esteem, social anxiety, and quality of life among the women with polycystic ovary syndrome. The Scientific World Journal. 2013;2013:851815.

[21] Johansson J, Redman L, Veldhuis PP, Sazonova A, Labrie F, Holm G, et al. Acupuncture for ovulation induction in polycystic ovary syndrome: a randomized controlled trial. American Journal of Physiology Endocrinology and Metabolism. 2013;304:E934-43.

[22] Jedel E, Labrie F, Oden A, Holm G, Nilsson L, Janson PO, et al. Impact of electro-acupuncture and physical exercise on hyperandrogenism and oligo/amenorrhea in women with polycystic ovary syndrome: a randomized controlled trial. American Journal of Physiology Endocrinology and Metabolism. 2011;300:E37-45.

[23] Barry JA, Kuczmierczyk AR, Hardiman PJ. Anxiety and depression in polycystic ovary syndrome: a systematic review and meta-analysis. Human Reproduction. 2011;26:2442-51.

[24] Deeks AA, Gibson-Helm ME, Paul E, Teede HJ. Is having polycystic ovary syndrome a predictor of poor psychological function including anxiety and depression? Human Reproduction. 2011;26:1399-407. 


\section{Figure Legends}

Figure 1. The process of study selection for the systematic review with meta-analysis of the effects of acupuncture on polycystic ovary syndrome. 
Citations from electronic searches and from examination of reference lists of primary and review articles: $n=173$

excluded after screening titles and/or abstracts: $n=98$

Full manuscripts retrieved for detailed evaluation: $n=75$

\begin{tabular}{|l}
$\mid \begin{array}{l}\text { Articles excluded with reasons: } 66 \\
\text { Reviews/commentaries }=27 \\
\text { Prospective case-controlled studies }=7 \\
\text { Other observational design }=7 \\
\text { Inappropriate study design }=13 \\
\text { PCOS-related data not collected }=12\end{array}$ \\
\hline The controlled trials with usable information for meta-analysis $(\mathrm{n}=9)$
\end{tabular} 
Figure 2. Meta-analysis of the studies evaluating the effects of acupuncture on the recovery of menstrual cycles (A), body mass index (B), fasting insulin (C) and plasma glucose (D) of the women with polycystic ovary syndrome.

OR, odds ratio; SMD, standardized mean difference; CI, confidence interval. 


\section{A}

Study

ID

OR $(95 \% \mathrm{Cl})$

Weight

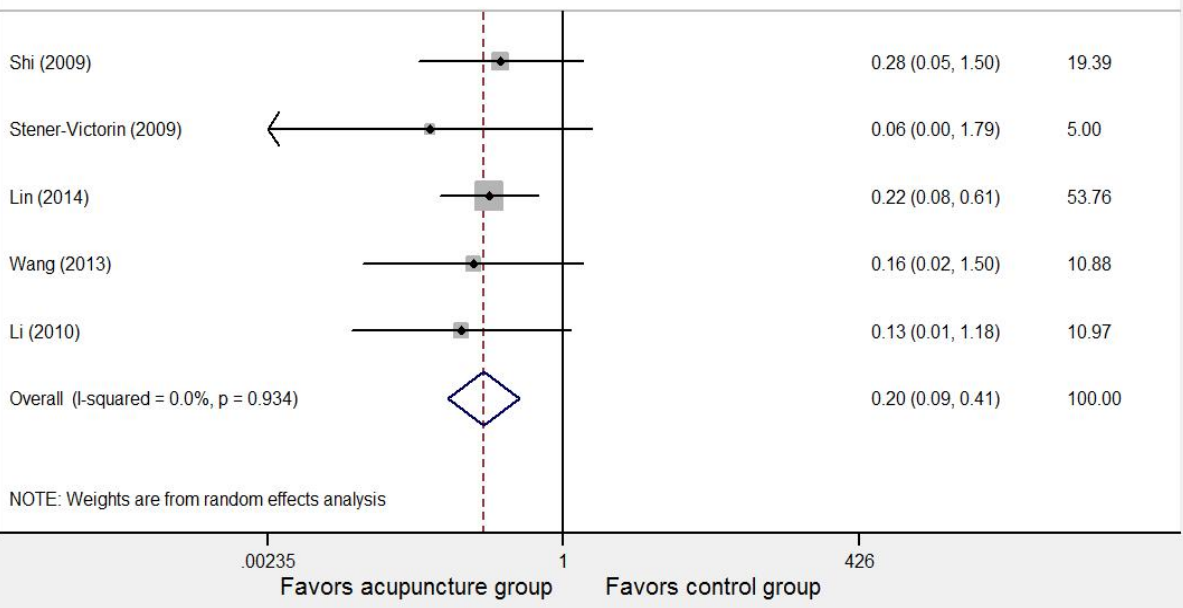

\section{C}

Study

ID

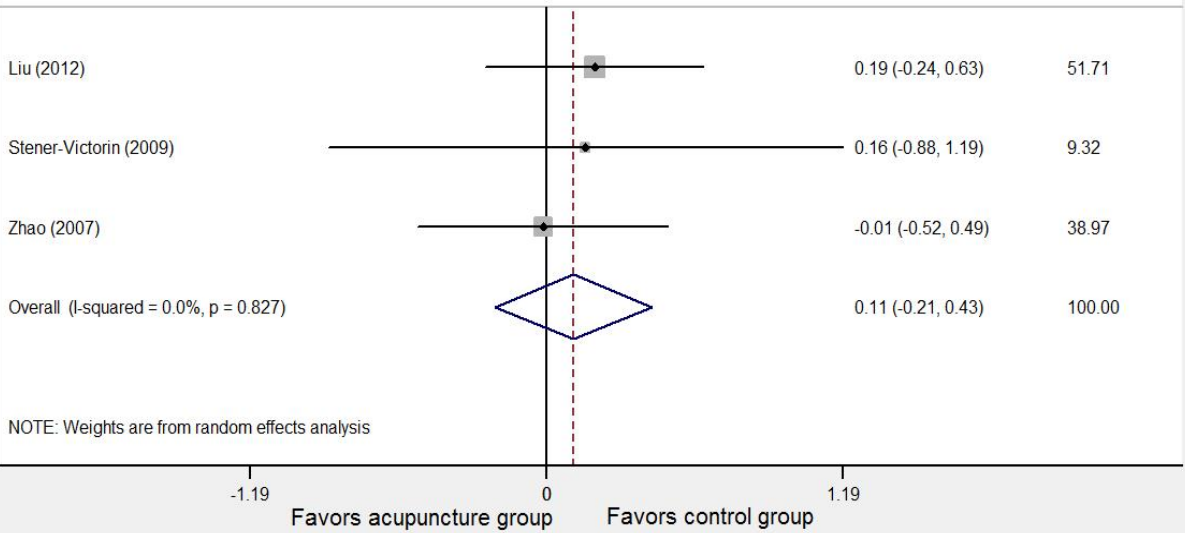

B

Study

SMD $(95 \% \mathrm{Cl})$

Weight



\section{D}

\section{Study}

ID

$\operatorname{SMD}(95 \% \mathrm{Cl})$

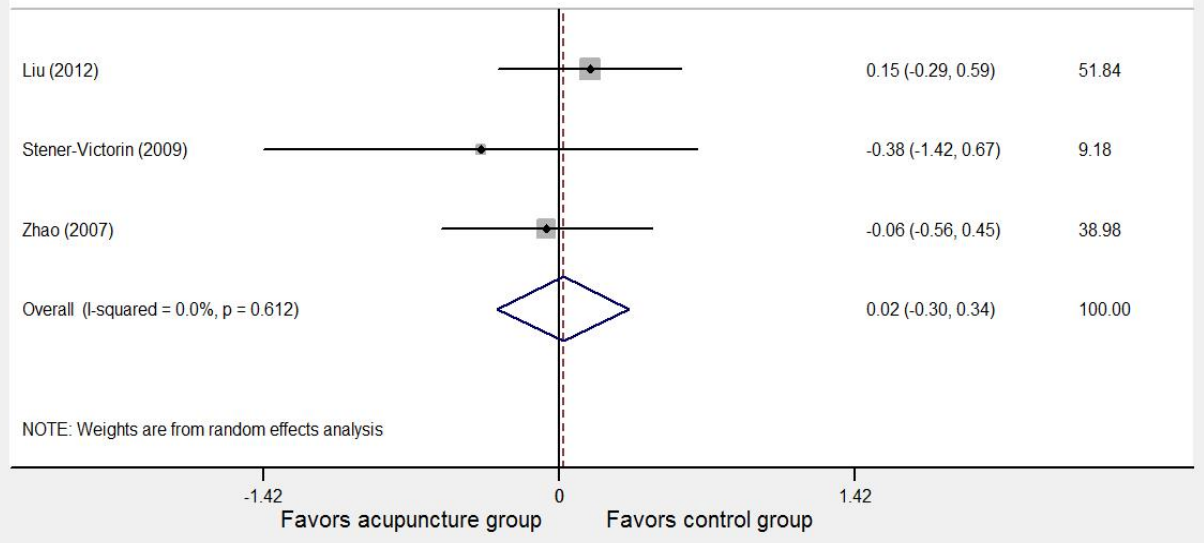


Figure 3. Meta-analysis of the studies evaluating the effects of acupuncture on the serum luteinizing hormone (A), follicle stimulating hormone (B) and the ratio of the serum luteinizing hormone levels to serum follicle stimulating hormone levels $(\mathrm{C})$ of the women with polycystic ovary syndrome.

SMD, standardized mean difference; CI, confidence interval. 


\section{A}

ID

SMD $(95 \% \mathrm{Cl})$

Weight

ID
SMD $(95 \% \mathrm{Cl})$

Weight

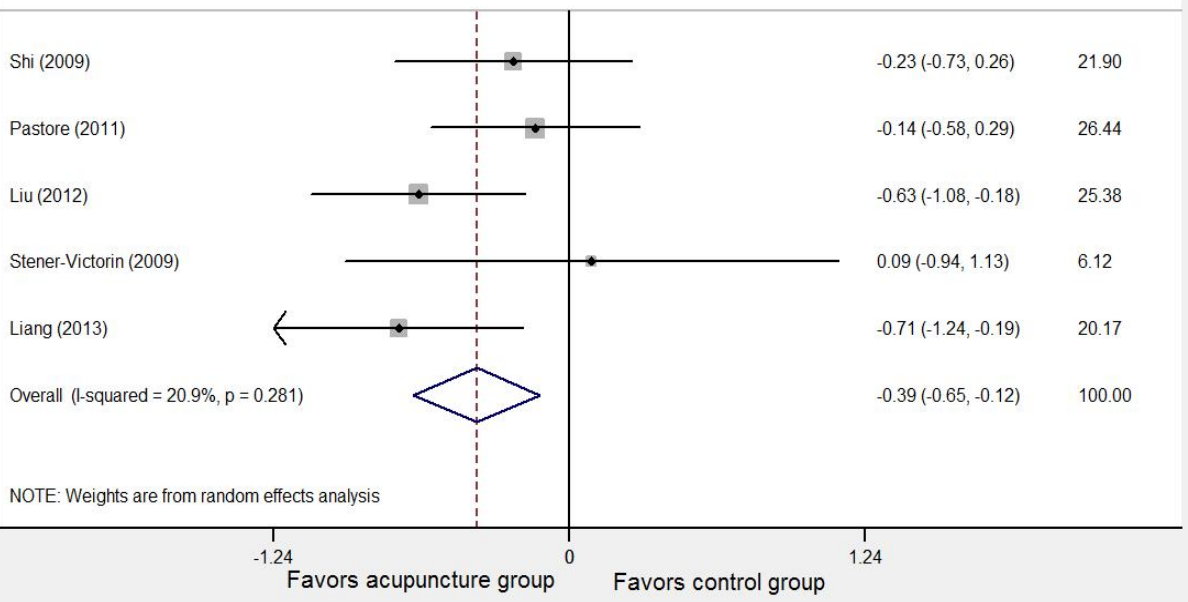

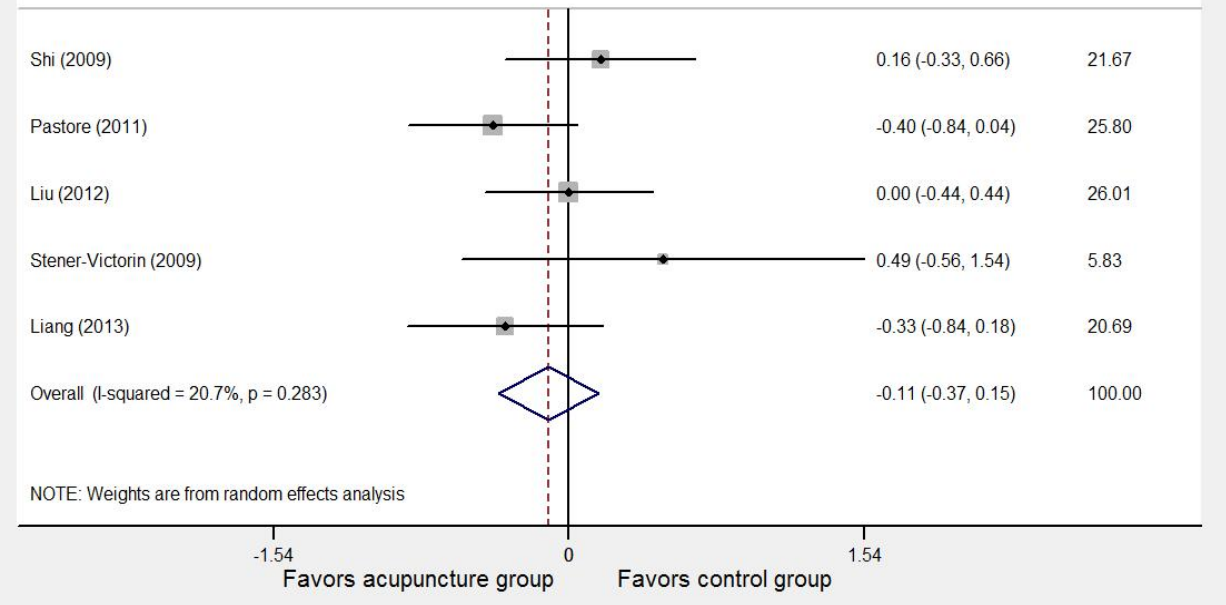

\section{C \\ Stud}

ID
B

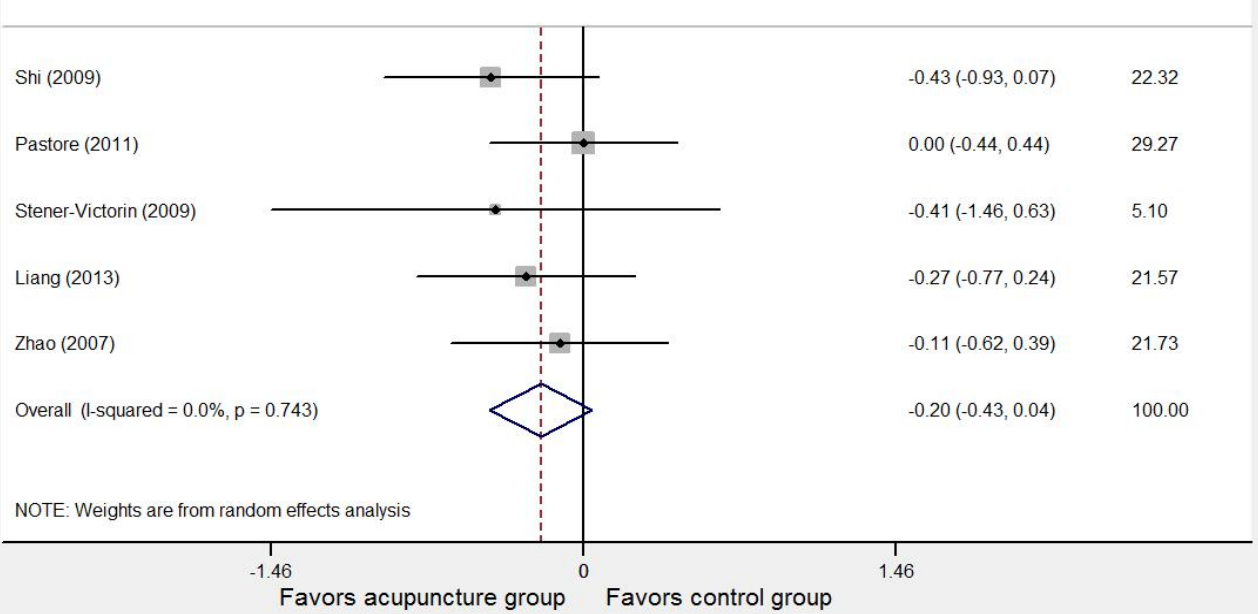


Table 1. Quality evaluation of the studies

\begin{tabular}{|c|c|c|c|c|c|c|c|c|c|c|c|c|}
\hline Study & $\begin{array}{l}\text { Description } \\
\text { of } \\
\text { information } \\
\text { of ethical } \\
\text { approval }\end{array}$ & $\begin{array}{l}\text { Randomization } \\
\text { Method }\end{array}$ & $\begin{array}{l}\text { Single- } \\
\text { /Multi- } \\
\text { centre }\end{array}$ & $\begin{array}{l}\text { Concealment } \\
\text { of allocation }\end{array}$ & $\begin{array}{l}\text { Placebo } \\
\text { Interventio } \\
\mathrm{n}\end{array}$ & $\begin{array}{l}\text { Blindin } \\
\mathrm{g}\end{array}$ & $\begin{array}{l}\text { Comparabilit } \\
\text { y } \\
\text { at baseline }\end{array}$ & $\begin{array}{l}\text { Sample size } \\
\text { calculation }\end{array}$ & $\begin{array}{l}\text { Acupuncture } \\
\text { practitioner }\end{array}$ & $\begin{array}{l}\text { Description } \\
\text { of adverse } \\
\text { effects }\end{array}$ & $\begin{array}{l}\text { Follow- } \\
\text { up }\end{array}$ & $\begin{array}{l}\text { Adherence } \\
\text { to STRICTA }\end{array}$ \\
\hline Shi et al. [14] & Yes & $\begin{array}{l}\text { A random } \\
\text { number table }\end{array}$ & Single & ND & No & No & Yes & ND & ND & No & No & No \\
\hline $\begin{array}{l}\text { Stener-Victorin } \\
\text { et al. [13] }\end{array}$ & Yes & $\begin{array}{l}\text { Computerized } \\
\text { randomization }\end{array}$ & Single & Adequate & No & Yes & Yes & ND & $\begin{array}{l}\text { Acupuncturis } \\
\mathrm{t}\end{array}$ & No & No & Yes \\
\hline $\begin{array}{l}\text { Pastore et al.; } \\
\text { Franasiak et al. } \\
{[10,11]}\end{array}$ & Yes & $\begin{array}{l}\text { A random } \\
\text { number } \\
\text { generator } \\
\text { program. }\end{array}$ & Single & Adequate & Yes & Yes & Yes & Yes & $\begin{array}{l}\text { Acupuncturis } \\
\mathrm{t}\end{array}$ & No & Yes & Yes \\
\hline Liu et al. [15] & No & ND & Single & ND & No & No & ND & ND & ND & No & No & No \\
\hline $\begin{array}{l}\text { Liang et al. [16, } \\
\text { 17] }\end{array}$ & Yes & ND & Single & ND & No & No & Yes & ND & ND & No & No & No \\
\hline Lin et al. [18] & No & ND & Single & ND & No & No & Yes & ND & ND & No & No & No \\
\hline Zhao et al. [19] & No & ND & Single & ND & No & No & ND & ND & ND & No & No & No \\
\hline Wang et al. [9] & No & $\begin{array}{l}\text { Sequence of } \\
\text { recruitment }\end{array}$ & Single & ND & No & No & Yes & ND & ND & No & No & No \\
\hline Li et al. [12] & No & $\begin{array}{l}\text { Sequence of } \\
\text { recruitment }\end{array}$ & Single & ND & No & No & Yes & ND & ND & No & No & No \\
\hline
\end{tabular}

Note: STRICTA: stands for the standards for reporting interventions in clinical trials of acupuncture; ND: not described 
Table 2. Summary of the studies

\begin{tabular}{|c|c|c|c|c|c|}
\hline Study & Participants & Country & Intervention & Control & Outcomes \\
\hline Shi et al. [14] & 63 women with PCOS & China & Acupuncture+ CMH & $\mathrm{CMH}$ & MF, FSH, LH, T \\
\hline Stener-Victorin et al. [13] & $\begin{array}{l}15 \text { women with PCOS without } \\
\text { known endocrine or neoplastic } \\
\text { causes of hyperandrogenemia }\end{array}$ & Sweden & Low-frequency EA & No intervention & $\begin{array}{l}\text { Muscle sympathetic nerve activity, MF, } \\
\text { Anthropometrics, hemodynamics, } \\
\text { hormones, insulin sensitivity, blood lipids }\end{array}$ \\
\hline $\begin{array}{l}\text { Pastore et al.; Franasiak et al. } \\
{[10,11]}\end{array}$ & $\begin{array}{l}84 \text { women with PCOS aged } \\
\text { 18-43 years without hormonal } \\
\text { intervention } 60 \text { days before } \\
\text { enrollment. }\end{array}$ & U.S.A. & EA & $\begin{array}{l}\text { Sham acupuncture with the } \\
\text { validated Park sham device }\end{array}$ & LH, FSH, OR, longitudinal AMH \\
\hline Liu et al. [15] & 80 women with PCOS & China & Acupuncture+ ECAT & ECAT & FSH, LH, T, E2, PRL, BMI, FINS,FPG \\
\hline Liang et al. $[16,17]$ & 60 infertile women with PCOS & China & Acupuncture+Gn & $\mathrm{Gn}$ & Mature follicle, FSH, LH, T, PRL, P, E 2 \\
\hline Lin et al. [18] & 84 women with PCOS & China & Acupuncture+ ECAT & ECAT & MF, ovarian volume, sex hormone levels \\
\hline Zhao et al. [19] & $\begin{array}{l}60 \text { obese women with PCOS } \\
\text { without hormonal intervention } \\
90 \text { days before enrollment. }\end{array}$ & China & $\begin{array}{l}\text { Acupuncture+ } \\
\text { Metformin }\end{array}$ & Metformin & $\begin{array}{l}\text { BMI, WHR, MF, LH/FSH, T, FINS, FPG, } \\
\text { HOMA-IR, TC, HDL-C, LDL-C }\end{array}$ \\
\hline Wang et al. [9] & $\begin{array}{l}40 \text { women with PCOS without } \\
\text { hormonal intervention } 180 \text { days } \\
\text { before enrollment. }\end{array}$ & China & Acupuncture $+\mathrm{CMH}$ & $\mathrm{CMH}$ & MF, CPR, BBT \\
\hline Li et al. [12] & 45 women with PCOS & China & Acupuncture+ $\mathrm{CMH}$ & $\mathrm{CMH}$ & MF(+), BBT, ovulation, hormones \\
\hline
\end{tabular}

Note: PCOS: polycystic ovary syndrome; CMH: Chinese medicinal herbs; MF: menstrual frequency; FSH: follicle stimulating hormone; LH: luteinizing hormone; T: testosterone; EA: electro-acupuncture; OR: ovulation rate; AMH: anti-m"ullerian hormone; ECAT: ethinylestradiol and cyproterone acetate tablets; E2: estradiol; PRL: prolactin; BMI: body mass index; FINS: fasting insulin; FPG: fasting plasma glucose; Gn: gonadotropin; P: progesterone; WHR: waist-hip ratio; HOMA-IR: homeostasis model assessment for insulin resistance; TC: total cholesterol; HDL-C: high-density lipoprotein cholesterol; LDL-C: low-density lipoprotein cholesterol; CPR: clinical pregnancy rate; BBT: basal body temperature. 